\title{
Lombalgias e Trabalho
}

Aparecida Mari Iguti ${ }^{1}$

Eduardo Luiz Hoehne ${ }^{2}$

\section{Occupational Low Back Pain}

'Docente do Departamento de Medicina Preventiva e Social da Faculdade de Ciências Médicas da UNICAMP.

${ }^{2}$ Estatístico do Departamento de Medicina Preventiva e Social, Faculdade de Ciências Médicas da UNICAMP.
Lombalgias apresentam altas taxas de prevalência em todos os setores de atividades econômicas sendo que em algumas, a gravidade das alterações levam ao afastamento e à incapacidade ao trabalho. Nos estudos observa-se a necessidade de avaliações e intervenções ergonômicas. Este é um texto de uma revisão das lombalgias e de seus fatores ocupacionais, considerando-se alguns aspectos metodológicos da avaliação da exposição aos fatores de risco; também pontuamos algumas dificuldades atuais, as necessidades e tendências de pesquisas neste tema.

Palavras chaves Lombalgia, Revisão, Fatores Ocupacionais.

Low Back Pains show high rates of existence in all sectors of economical activities which, in some cases, the high risk of alterations lead the worker to absence in workplace and definite work incompetence. In the studies it is observed the need of avaluations and ergonomic interventions. This is a text about the focus on Low Back Pain and its occupational factors; taking under consideration some methodologic aspects regarding the avaluation of the exposition to the risky factors; we also point out some actual problems, the needs and tendencies of research on this field.

Keywords Low Back Pain, Occupational Factors. 


\section{Introdução}

As doenças osteo músculo esqueléticas causam um impacto significativo sobre a população trabalhadora, pelo sofrimento físico e psíquico diretos, com repercussões sobre a vida social e econômica, além de gerar incapacidades e limitações no trabalho e na vida cotidiana. As lombalgias em particular, são responsáveis por 15 a $20 \%$ de todas as notificações de doenças e aproximadamente um quarto dos casos de invalidez prematura. As perdas que resultam para as economias nacionais são elevadas; os pagamentos de dias perdidos de trabalho, tratamentos e indenizações por invalidez, custam vários milhões de dólares. Nos Estados Unidos, em 1979 o pagamento de benefícios diretos por problemas lombares foi de mais de um bilhão de dólares; em 1984, o custo total foi de cerca de 16 bilhões e em 1990, entre 50 e 100 bilhões (GUO,1995). MACDONALD e colaboradores (1997) levantaram os dados de uma seguradora americana de benefícios trabalhistas, respondendo por $10 \%$ do mercado privado do país. O número de benefícios requeridos entre janeiro de 1986 a fevereiro de 1996 foi de 1.248 .659 de solicitações feitos por 1.051.262 indivíduos por lombalgia. Encontraram para o ano de 1990, 110.983 solicitações de benefícios por lombalgia; deste total, 15.308 (14\%) preencheu mais de uma solicitação de indenização (casos de lombalgia recorrente, sendo $77,2 \%$ homens e $22,7 \%$ mulheres). Dos casos considerados recorrentes, $81 \% \mathrm{fez}$ uma solicitação, $15 \%$ duas e $4 \%$ três ou mais num período de 6 anos. Este mesmo autor cita seis trabalhos de autores, para diferentes países, sobre as taxas de solicitação de benefício, por lombalgia recorrente: Quebec, 36,3\%, (1992), Estados Unidos, 18\%, (1986); 45,2\% (1993); Inglaterra, 44\%, (1996); Suécia, 31\% (1977).

WEBSTER \& SNOOK (1990) analisando os custos da seguradora Liberty Mutual Insurance, que detinha na época estudada $11 \%$ do mercado americano de compensação para trabalhadores, observaram que os custos totais de indenização para o ano de 1986 foram de 673.884.128 dólares, só para benefícios com lombalgias. Também notaram que nos 45 estados americanos onde o seguro atuava, a média do custo por caso foi de 6.807 dólares e a mediana de 391 dólares. Esta diferença indica que a variação deve-se ao fato que a distribuição de custos não é homogêneo, e que $25 \%$ dos casos consome $95 \%$ do total de custos, por hospitalização, cirurgias, contestações judiciais, incapacidade psicológica e longos períodos de afastamento do trabalho. Também na distribuição por estado, a variação de custos médios foi enorme, indo de 1.714 dólares no estado de Indiana a 18.668 dólares, no Estado de Lousiana.

Em 1993 na Grã Bretanha, segundo o Grupo Consultor de Normas Clínicas (CSAG) a prevalência de dor lombar crônica foi de 16,5 milhões de pessoas, sendo o custo anual para o Serviço Nacional de Saúde, de 480 milhões de libras esterlinas e o custo com benefícios previdenciários de 1400 milhões de libras esterlinas. O número de casos de incapacidade tem aumentado exponencialmente entre 1955 e 1994 (de 10 milhões para 75 milhões de dias de trabalho perdidos por ano).

Projeções de especialistas americanos e europeus indicam que 15 a $20 \%$ da população apresenta Dor Lombar Crônica (DLC) e que quase todos os adultos apresentam em algum momento de suas vidas, DLC de alguma intensidade. DLC não está restrita aos países desenvolvidos, tem sido descrita também em regiões rurais da Índia e Nepal. Estima-se que entre 5 a $10 \%$ dos indivíduos que apresentam dor lombar aguda, cronificam. Seu custo anual direto é de cerca de U\$20 bilhões e seu custo indireto, incluindo perda de rendimentos de U\$75 a 100 bilhões. Entre $70 \%$ a $90 \%$ dos custos corresponde ao pagamento dos 10\% de incapacitados pela doença. Em Israel, país com menos de 3 mithões de trabalhadores, gastam-se quase U\$1 bilhão em dias perdidos por absentismo por dor lombar. (BORKAN, 1995).

Alguns estudos são citados para destacar a prevalência anual de lombalgia em diferentes países. São: 14-16\% para população geral americana (1994); $20 \%$ para trabalhadores (UK) (1986); 10\% para população geral americana (1987); 8\% para trabalhadores dinamarqueses (1987); $20 \%$ para trabalhadores americanos (1989); $18 \%$, trabaIhadores americanos (1995); 35-37\%, população geral, Reino Unido, (1995). Somente $10 \%$ dos que apresentam os episódios solicitam benefícios. A taxa de incidência de 
compensação por lesões de coluna varia de 0,3 a 3,3 casos por 100 trabalhadores/ano. Entretanto, os que se tornam crônicos apresentam custos elevados nos benefícios (HALES \& BERNARD, 1996).

GUO (1995) cita autores de distintos países com suas estimativas de frequência: $17,6 \%$ de prevalência, em estudo 50 estados, EUA (1988); 0,87 solicitação de benefício seguritário por 100 trabalhadores, estudo realizado em 26 estados, USA, ano de 1979; $20 \%$ de prevalência, nos 50 estados americanos, para o ano de 1973; uma prevalência de $20 \%$ em homens no Reino Unido, ano de 1986; uma prevalência de $8 \%$ na Dinamarca, ano de 1982; uma incidência de 0,29\%, na Suécia, ano de 1980; 3,29 solicitações por 100 trabalhadores na Nova Zelândia, ano de 1984, com incidência de 1,7\% no Quebec, Canadá, ano de 1981 ; prevalência de 1,37\%, no Quebec, Canadá, ano de 1981. LEUNG e colaboradores (1999) citam prevalência de 39\% (vida toda) e $21 \%$ a frequência anual, para população de Hong Kong. Estes dados mostram a grande variação de estimativas de frequência para diversas populações, e as dificuldades metodológicas em função das diferentes fontes de dados e cálculos; de toda forma, os números de portadores de lombalgia têm sido expressivos.

SYMONDS e colaboradores (1996) referem, a partir das estatísticas oficiais, que em 1993, foram perdidos 81 milhões de dias de trabalho, devido às lombalgias, um aumento de 21\% em relação ao ano de 1992. Nos Estados Unidos a dor lombar é a causa mais freqüentemente responsável por limitação de atividade em trabalhadores com menos de 45 anos de idade (KELSEY et al.,1990)

US Health Interview Survey, em publicação de 1973 (KELSEY, 1990) aponta como a causa mais frequente de condição crônica levando à incapacidade, para indivíduos de menos de 45 anos, os problemas de coluna vertebral; é a terceira causa de incapacidade, depois de doenças cardíacas e artrites / reumatismo, em pessoas na faixa etária entre 45-64 anos. Na lista da NIOSH (1988) das 10 principais doenças e lesões relacionadas ao trabalho, as lesões músculo esqueléticas e que incluem as alterações de coluna cervical, dorsal lombar, membros superiores e inferiores, fenômeno de Raynaud de origem ocupacional ocupavam o segun- do lugar, em termos de freqüência.

WADDELL \& BURTON (2001) em estudo sobre as lombalgias no trabalho, consideram que existem fortes evidências epidemiológicas que 60 a $80 \%$ dos adultos experimentam lombalgia em algum momento de suas vidas, e frequentemente é persistente ou recorrente. É uma das mais comuns razões para a procura de cuidados médicos e para absenteísmo no trabalho por motivos de saúde.

BEHRENS e colaboradores (1994) estimaram uma prevalência de 2,5\% da população trabalhadora americana ou seja, 2,62 mithões de indivíduos apresentaram lombalgia nos 12 meses precedentes ao inquérito. $A$ prevalência geral de lombalgia devido a atividades repetitivas foi estimada em $4,5 \%$, o que representa 4,75 milhões de trabalhadores.

KATZ \& RONDINELLI (1998) consideram, a partir de dados de literatura, que a prevalência de incapacidade por problemas lombares entre os países do ocidente varia bastante. Como exemplo estimam que a prevalência para os Estados Unidos, Canadá e Reino Unido é de aproximadamente $2 \%$, Alemanha Ocidental e Holanda de $4 \%$ e Suécia de 8\%. Dados da Suécia mostram que $60 \%$ dos indivíduos já passou pela experiência da dor, sendo que destes $16 \%$ apresentou sintomas entre três e seis meses, com incapacidade e $4 \%$ apresentou incapacidade por mais de seis meses.

SHELERUD (1998) utilizando dados de KLEIN (1984) levanta taxas de solicitação de benefícios por problemas de coluna, em 26 estados americanos e por ocupação, e encontra $12,3 \%$ para uma mistura de trabaIhadores, 11,1\% para lixeiros, 9,3\% para trabalhadores de mercados, 5,6\% para mecânicos (de variados setores), 3,6\% de auxiliares de enfermagem, 3,4\% trabalhadores não especificados, 3,4\% para trabalhadores manuais, $3,3 \%$ para trabalhos pesados, $3,3 \%$ para atendentes de enfermagem, 2,8\% para trabalhadores da construção civil. O mesmo autor selecionou dados de incidência e prevalência de lombalgias na vida dos trabalhadores e encontrou entre autores, incidências que variavam entre $51,4 \%$ a 69,9\% e prevalências atuais que variavam entre 12 a $30,2 \%$.

ABENHAIM e colaboradores (1988) acompanharam 2342 indivíduos que apresentaram algias de coluna no Quebec, Canadá; 
a taxa de recorrência foi de $22 \%$ no primeiro e $36,5 \%$ no terceiro ano. No primeiro ano de seguimento houve casos de até seis episódios álgicos; no terceiro, de até nove episódios. Os homens apresentaram maior possibilidade de recorrência, mas entre os recorrentes, a média total foi semelhante entre homens e mulheres. A idade mostrou "efeito protetor" sobre a probabilidade de recorrência.

Estudos epidemiológicos feitos nos países baixos, escandinavos e americanos, indicam que na faixa de 20 a 65 anos, de 51 a $81 \%$ dos interrogados apresentaram ao menos um episódio doloroso; 2 a $5 \%$ da população geral de clínica medica consulta médicos por lombalgia. A incidência anual do primeiro episódio doloroso entretanto é mal conhecido; pode chegar a $10 \%$ antes dos 30 anos, decrescendo progressivamente; a evolução espontânea da sintomatologia é favorável na grande parte dos casos: 90 a $95 \%$ dos que apresentaram incapacidade, retornam ao trabalho nos três meses que se seguem ao episódio.

Um estudo feito na Dinamarca, por BIERING-SORENSEN (1984), mostrou que durante a vida laboral, homens e mulheres relatam lombalgia com frequências semelhantes; entretanto as indenizações referidas por acidentes de trabalho são muito mais frequentes para homens.

HYYTIAINEN (1994) da Finlândia, encontrou entre trabalhadores administrativos $(n=170)$ e de produção $(n=504)$ de duas indústrias, naval e de peças de ventiladores, $73 \%$ e $71 \%$ de lombalgia referida em algum momento de suas vidas. Entre os de escritório, $23 \%$ relataram quadros álgicos contínuos ou recorrentes e $28 \%$ dos de produção. 4 a $5 \%$ da população sofre a cada ano de lesões discais. Para uma comparação, a gripe, englobando outras patologias respiratórias agudas, atinge cerca de $6 \%$ da população a cada ano. As lesões discais encontram-se entre as mais frequentes causas de doenças. Mesmo em relação às alterações de coluna mais tardias, como a espondilose - as lesões discais desempenham um papel importante. As algias de coluna representaram de 19 a $25 \%$ das solicitações de indenização entre os trabaIhadores americanos. A maioria das hérnias de disco ocorrem entre 20 e 64 anos de idade, em pico de frequência entre 25 e 45 anos de idade; as que se submetem a cirurgias são mais frequentes na faixa de 30 a 39 anos de idade. Pelo menos um em cada mil trabalhadores inseridos em atividades industriais desenvolve anualmente, hérnia de disco, com idade entre 20 e 64 anos de idade, em países como Inglaterra, Copenhague (KELSEY, 1990)

A freqüência de hérnias de disco nos Estados Unidos é desconhecida. Segundo a US Health Interview Survey, 1\% dos entrevistados na faixa de 17-64 anos referiu-se a hérnia de disco. A hérnia de disco é a segunda causa de incapacidade, em indivíduos com menos de 40 anos, perdendo somente para a esquizofrenia; para todas as idades, é a terceira causa, após as doenças cardíacas e as osteo-artrites. Além das degenerações discais, diferentes patologias congênitas ou adquiridas podem causar algias dorso-lombares e serem agravadas por um trabalho físico cansativo e excessivo. Por exemplo, a doença de Schewermann piora na puberdade, após sobrecarga da coluna (BEHRENS, 1994)

A partir de estudo populacional $(n=7217)$ na Finlândia, HELIOVAARA e colaboradores (1987) estimaram que 127.000 finlandeses com mais de 30 anos eram portadores de síndrome discal lombar; 65.000 destes necessitariam de controle médico e 9.000 estariam com cuidados de saúde inadequados. No estudo conduzido pelos autores, a prevalência de síndrome discal lombar foi de $5,1 \%$ entre homens e de 3,7\% entre mulheres. A proporção da população requerente de cuidados foi de $2,4 \%$. Entre os indivíduos com diagnóstico e os com suspeita, $84 \%$ necessitaria de assistência médica.

Na Dinamarca, GYNTELBERG (1974), avaliando 4753 trabalhadores encontrou lombalgia referida no decorrer do ano em $26 \%$ deles, sendo que $11 \%$ apresentou irradiação para membros inferiores, $8 \%$ teve que ficar de repouso no leito e ausente do trabalho; $8 \%$ submeteu-se a tratamento para as dores.

Num estudo conduzido por VALLFORS (1985) na Suécia, exames clínicos cuidadosos não revelaram nenhum sinal objetivo para um terço dos pacientes com sintomas agudos e subagudos, com afastamento do trabalho até um mês e para dois terços dos que apresentavam sintomas crônicos, com afastamento de até três meses. No grupo com sintomas agudos e subagudos sem sinais objetivos, a taxa de recuperação foi mais rápida quando 
comparado com o grupo que apresentava sinais objetivos. (RIIHIMAKI, 1991). HALES \& BERNARD (1996) consideram que felizmente a maioria dos episódios são autolimitados. Cerca de $90 \%$ apresenta recuperação espontânea e em até um mês retorna às atividades normais. $20 \%$ dos lombálgicos procura serviço médico.

LEUNG e colaboradores (1999) estudaram uma população chinesa, de Hong Kong, portadora de lombalgia e que se submeteram a tratamento fisioterápico. Estes pacientes foram selecionados em função da provável origem mecânica do episódio álgico, sem sintomas neurológicos associados. Destes, $82,7 \%$ apresentava história de episódios recorrentes; somente em $17,3 \%$, tratava-se do primeiro episódio álgico. Do total, $49 \%$ iniciou o tratamento fisioterápico em três semanas ou menos após o início do episódio e 51 \% com mais de três semanas.

\section{Ocupações e atividades de risco para lombalgias}

GUO e colaboradores (1995) apresentam as quinze profissões masculinas com os maiores índices de prevalência nos Estados Unidos, em ocupações com mais de 349.000 homens expostos. São elas: trabalhadores da construção civil, carpinteiros, operadores de tratores e máquinas industriais pesadas; mecânicos de automóveis; agricultores (exceto de horticultura); supervisores de construção; soldadores, encanadores e caldeireiros; reparadores de máquinas industriais; estoquistas; motoristas de caminhões pesados; trabalhadores da agricultura; jardineiros; motoristas de caminhões leves; operadores de soldagem e de oxicorte; operários, exceto da construção. Entre as mulheres, as quinze profissões, com mais de 290.000, incluem: auxiliares e atendentes de enfermagem; técnicas de enfermagem; domésticas; porteiras e auxiliares de limpeza; operárias; cabeleireiras e cosmetologistas; inspetores de produção, controladoras e examinadoras de qualidade; designers; auxiliares de saúde (não enfermagem); garçons e garçonetes; enfermeiras; cozinheiras; operadoras de tecelagem; professoras; caixas.

A NIOSH (1992) citada por GUO (1995) aponta os estudos mais frequentes, de atividades relacionadas com lombalgia, sem or- dem classificatória. São os auxiliares, atendentes de enfermagem; enfermeiras; técnicas de enfermagem; operadores de máquinas em minas; trabalhadores da construção civil; pilotos de aviação e navegadores; motoristas de caminhões pesados; operadores de máquinas e equipamentos industriais; agricultores (não horticultura); motoristas de caminhões leves; condutores de ônibus.

BEHRENS e colaboradores (1994), utilizando os dados do National Health Interview Survey de 1988, com 44.233 indivíduos entrevistados nos Estados Unidos, encontraram diferenças acentuadas entre grupos ocupacionais, com aumento da prevalência de lombalgia devido a lesões, em motoristas de caminhões $(6,7 \%)$ e entre mecânicos de manutenção de equipamentos pesados (10,5\%).

SHELERUD (1998) encontrou na literatura dados sobre o risco relativo de herniação discal e ciática somados, para diferentes setores de atividade econômica; considerou os profissionais e trabalhadores de escritório como grupo de referência ( $R R=1$ ) e assim temos $R R=4,6$ para motoristas de veículos pesados $(p<0,001), R R=4,2$ para operadores de máquinas/metal ( $p<0,001), R R=3,2$ para operadores de processos químicos/papel $(p<0,01), R R=3,1$ para trabalhadores madeira $(p<0,05), R R=3,1$ para trabalhadores da construção civil $(p<0,01), R R=2,5$ para agricultores e assemelhados $(p<0,05)$.

Em estudo realizado na Finlândia observou-se que entre homens com maiores riscos de hospitalização por hérnia de disco encontram-se os motoristas de caminhão e trabaIhadores de indústria, particularmente entre os de metais e máquinas; entre mulheres, algumas atividades industriais e pessoal de enfermagem. Os motoristas apresentaram as mais altas taxas de recorrência e as enfermeiras, o maior número médio de recorrências; estas duas profissões tiveram um excesso de casos estatisticamente significativo, após o controle de outras variáveis. (KELSEY, 1990)

TSAl e colaboradores (1992) levantaram dados de lesões músculo esqueléticas em 10.350 empregados da Shell Petróleo, nos Estados Unidos, entre os anos de 1987 a 1989. O risco relativo de lesões lombares foi maior entre fumantes ( $R R=1,54, p<0,01$ ) e os que apresentavam sobrepeso (IMC acima de $20 \%$ do predito), ( $R R=1,42, p<0,01)$. 
Os empregados com atividades físicas de maior demanda, basicamente os de manutenção, apresentaram maior risco relativo para lombalgias $(R R=1,57 p<0,01)$ e para outras lesões músculo esqueléticas ( $R R=1,35$ $\mathrm{p}<0,01)$

LEIGH (1989) apresenta em um inquérito de prevalência de dores lombares/coluna em 1414 trabalhadores em "tempo integral" (com mais de vinte horas semanais) nos Estados Unidos, em amostragem nacional, uma correlação positiva significativa para trabalhadores rurais, de serviços braçais; também com os de baixo nível de escolaridade; trabalhadores com componente de esforço físico; idade entre 50 e 64 anos e os fumantes.

GRANIER e colaboradores (1998) em uma amostra de 464 trabalhadores num universo de 2587 funcionários públicos franceses encontraram uma prevalência de lombalgia de $42,2 \%$ e entre os que apresentavam história anterior, este número subiu para $51,7 \%$. A frequência de casos tende a aumentar com a idade, até a faixa de 50 anos e reduzir a seguir. $O$ primeiro episódio álgico tende a ocorrer em torno de trinta anos de idade; a incidência anual, excluídos os que apresentam antecedentes, é de $22,6 \%$ e diminui com a idade. Entre os que referiram lombalgia $52,4 \%$ relatou sobrecarga física, $57,6 \%$ fadiga no trabalho e $51 \%$, conflitos no trabatho, sendo que as freqüências entre os que não apresentaram dores foram menores para todas as características de trabalho.

FEYER e colaboradores (1992) descrevem as principais localizações de dores lombares, para três grupos estudados na Austrália, que estão no quadro 1, que citamos a seguir:

Quadro 1 Distribuição das principais localizações de dores na coluna.

\begin{tabular}{|l|c|c|c|}
\hline localização & enfermeiros (\%) & carteiros (\%) & pacientes lombálgicos (\%) \\
\hline L5-S1 & 84 & 68 & 73 \\
\hline glúteo D & 67 & 59 & 76 \\
\hline Sacro cóccix & 67 & 70 & 87 \\
\hline glúteo E & 60 & 57 & 73 \\
\hline Total & 198 & 187 & 45 \\
\hline
\end{tabular}

FEUER (1992)

Nos três grupos, a gravidade da dor foi um importante fator preditivo para incapacidade referida. Entre os enfermeiros, a gravidade da dor foi fator preditivo para o tempo de afastamento do trabalho, enquanto entre carteiros, o fato de sentir-se incapacitado foi o fator preditivo do tempo de afastamento do trabalho.

DERRIENNIC (1993) avaliando a evolução de alterações osteo-articulares em função de histórico de atividades físicas intensas, em um estudo na região parisiense, fez uma pesquisa domiciliar de 627 aposentados que haviam contribuído para um mesmo fundo de pensão, no período de 1982-83 e em 1987 88 , em 464 dos mesmos aposentados. Foram considerados como expostos a fatores ocupacionais os indivíduos que haviam trabalhado por mais de 10 anos em atividades de mesma natureza. As alterações osteo-articulares foram avaliadas pela presença de dor com ou sem restrição do movimento articular, durante os seis meses ou mais anteriores à entrevista. Entre os entrevistados nos dois períodos, houve um aumento da frequência de dores osteo-articulares, de $52 \%$ para $65 \%$, entre os homens e de $72 \%$ para $82 \%$ entre as mulheres. Em ambos os sexos, os que haviam trabalhado em atividades pesadas, tais como transporte de cargas (carregar pesos) e posições cansativas, as frequências eram significativamente maiores. Embora existam fatores de confundimento, como a idade na primeira entrevista, o fato de viver sozinho, ter sido trabalhador braçal, ter problemas cardio-respiratórios e fumar, este estudo sugere que condições de trabalho constituemse em importante causa de distúrbios osteoarticulares, presentes após o término da vida laboral ativa.

HADLER (1982) cita estudo de MAGORA (1974) de 3.316 trabalhadores em diversas 
profissões, cuja prevalência de dor lombar foi de $22 \%$ para trabalhadores de indústria pesada, $17 \%$ para enfermeiros, $15 \%$ para agricultores, 14\% para indústrias "leves", $12 \%$ para motoristas de ônibus, e 10\% para empregados de escritório e correios.

GAUDEMARIS e colaboradores (1986) levantando dados, em inquérito feito por médicos do trabalho por ocasião dos exames periódicos, de riscos de desenvolver lombalgias em 1812 trabalhadores franceses e encontraram que atendentes de enfermagem $(R R=2,24)$ e trabalhadores de manutenção industrial $(R R=1,54)$ apresentaram riscos relativos mais elevados, quando comparados com empregados de escritório. Em análise de multivariância encontrou-se que as 'atividades de manutenção industrial pesada ou leve, as posturas de coluna viciosa, o trabalho em posição de pé sem possibilidade de sentar, a exposição a vibrações e às intempéries, foram correlacionadas com o risco aumentado de desenvolver episódios de lombalgia.

No Brasil, FINOCCHIARO \& ASSAF (1976) estudando 6500 perícias médico-judiciais, observaram que para a construção civil, de 1669 casos relacionados ao trabaIho, 1290 tratavam-se de afeções da coluna vertebral e entre elas, 1262, eram de lombalgias. As causas mais frequentes imputadas foram, por ordem decrescente: esforços físicos (31\%); esforços em flexão (25\%); quedas (18\%); traumas diretos (15\%); sobrecargas $(5,3 \%)$. Entre as profissões mais atingidas, encontravam-se os pedreiros, serventes, carpinteiros, armadores e os ajudantes gerais.

As maiores prevalências estimadas foram para mecânicos de reparos de veículos, máquinas e equipamentos pesados $(10,5 \%)$, seguido de operadores de extração, mineração e movimentação de cargas $(10,4 \%)$ e pessoal da construção civil $(10,1 \%)$. (BEHRENS et al, 1994).

CHIRON (1983) em documento do Instituto de Pesquisas sobre Segurança do TrabaIho (INRS) francês, estuda os problemas reumáticos em motoristas de carga pesada; cita vários trabalhos onde a frequência de problemas de coluna vertebral são altos, como os de GRUBER, publicado em 1974, sobre 1000 motoristas americanos, acompanhados num período de 10 anos, em relação ao estado de saúde. Um dos aspectos significati- vos é que as algias se relacionam mais com o tempo trabalhado e menos com a idade em si. Comparando-se com empregados de escritório, encontrou entre os grupos de doenças, um aumento significativo de doenças osteo-articulares. A estimativa de risco é que 11 em cada 100 motoristas desenvolvam doenças do sistema osteo músculo esquelético após 10 anos de trabalho. Em outro estudo compara com os motoristas de ônibus e observa diferenças nos tipos de afeções encontradas; nos motoristas de caminhão, as síndromes dolorosas e hérnias de disco são as mais frequentes, enquanto nos de ônibus, as afeções sacroilíacas e rigidez articulares. Também os tratoristas constituem-se em outro grupo sob risco ocupacional para as doenças músculo esqueléticas.

VIGNON \& MASSET (1979) avaliaram de 10 a 16 anos após, 51 lombálgicos que se internaram em hospital por acidente de trabalho, ocorridos entre 1962 e 1968 e que preenchiam os seguintes critérios: serem homens entre 30 a 45 anos de idade, trabalhadores manuais ( 12 metalúrgicos, 10 da manutenção, 9 da construção civil, 6 ferroviários, ou da eletricidade, 5 mineiros e 9 diversos), todos acidentados do trabalho, sem patologia prévia de coluna, sem alterações. $90 \%$ após 10 anos ainda sofria de dores, $57 \%$ teve que mudar de posto de trabalho e $7,8 \%$ foi aposentado. Os autores referem-se ao trabalho de CHAQUAT (1978) onde compara com os trabalhadores ditos intelectuais, e que nestes, de 93, somente dois tiveram incapacidade com impactos sobre sua vida financeira.

ABENHAIM e colaboradores (1988) observaram que as profissões onde os episódios recorrentes de dores de coluna mais frequentes são de motoristas $(42,1 \%)$, enfermeiras $(39,6 \%)$, trabalhadores braçais $(36,6 \%)$, mineiros $(36,4 \%)$ e trabalhadores de escritório $(28,6 \%)$. As maiores taxas de risco relativo, comparadas com os de trabalhadores de escritório foram para enfermeiras ( $R R=2,62$, $95 \%, I C=1,80-3,82$ ) e motoristas ( $R R=1,64$, $95 \% \mathrm{IC}=1,13-2,37)$.

KNIBBE \& FIELE (1996) realizaram um estudo descritivo com pessoal de enfermagem na Holanda e observaram que comparando a prevalência de lombalgias entre pessoal de enfermagem, que auxiliares e atendentes constituíam-se em grupo sob maior risco, por realizar tarefas diretas e cotidianas como trans- 
portar, carregar, erguer pacientes. Observaram entretanto um segundo grupo que também apresenta risco, são as jovens estudantes, talvez pelo fato de serem iniciantes, e que sua inexperiência contribua para que nesta faixa haja uma maior prevalência. Outros dois subgrupos são de pessoal de enfermagem domiciliar, pelas condições de menor infra-estrutura e as de geriatria, pela alta frequência de transferência de pacientes e de fatores não previstos que ocorrem com maior frequência.

STUBBS e colaboradores (1983) estudaram 3.912 enfermeiras na Inglaterra, e observaram que cada um em seis casos de lombalgia era atribuído à transferência de pacientes. Deste total, 15,3\% relatou um episódio álgico, 14,8\% dois episódios, 13,2\% três, $8,5 \%$ quatro episódios separados $3,4 \%$ cinco, $1,9 \%$, seis e $43,1 \%$ sete ou mais episódios.

LEIGHTON \& REILLY (1995) aplicaram questionário em 1134 trabalhadores de enfermagem e em 315 controles e as prevalências anuais foram respectivamente de $58,8 \%$ e $57,8 \%$. Para os dois grupos comparados não foram encontradas diferenças significativas; entretanto ao se detalhar para distintos subgrupos, em distintas especialidades, encontramos diferenças significativas. Por exemplo entre os trabalhadores de ortopedia $71 \%$ apresentou lombalgia no ano anterior à entrevista. Também enfermeiras de clínica médica $(68,9 \%)$, da comunitária $(62,5 \%)$, das unidades intensivas $(60,9 \%)$ e as de geriatria $(60,7 \%)$ tiveram taxas mais elevadas. Entre os fatores desencadeadores das algias, quase a metade recordou-se de um incidente precipitador ou agravante; em dois terços destas, o incidente ocorreu durante movimentação de pacientes. Listando as principais atividades implicadas nas lombalgias temos posicionando o paciente na cama $(47,7 \%)$, movendo paciente da cama $(23,4 \%)$, movendo paciente de cadeira $(13,1 \%)$, movendo paciente de banho $(4,5 \%)$, movendo paciente de toilete $(4,5 \%)$, movendo paciente do chão $(3,6 \%)$ e outros procedimentos $(3,2 \%)$. Tarefas como inclinar-se sobre o paciente, erguer e carregar pacientes e permanecer longo tempo de pé foram indicados por $25 \%$ como 'sempre' e 'frequentemente' associados com lombalgia.

HARBER e colaboradores (1985) no inquérito envolvendo 550 enfermeiros em hospital terciário da Califórnia, observaram que as principais atividades relatadas pelos trabalhadores como precipitadores ou agravantes de lombalgia são erguer paciente de leito $(48 \%)$, ajudar paciente a sair do leito $(30 \%)$, movimentar leitos (27\%), nenhum (25\% - em uma lista de 15 escolhas), erguer paciente para uma maca $(22 \%)$ carregar equipamento pesando mais de 30 libras (10\%). Embora a frequência de sintomas álgicos seja elevado, $52 \%$ do total, somente $4 \%$ dos que responderam foram hospitalizados e $2 \%$ relataram cirurgia devido a esse quadro. $3 \% \mathrm{mu}$ daram de trabalho e $23 \%$ relatou ter procurado serviço médico pelo menos uma vez na vida por causa de lombalgia.

\section{Indicadores de risco para lombalgias}

RIIHIMAKI, (1991) cita HILDEBRAND (1987) numa revisão de estudos epidemiológicos e 24 diferentes fatores laborais são considerados, entre eles, os trabalhos físicos pesados, posturas no trabalho em geral; trabalho estático; posturas estáticas em geral como permanecer sentado por tempo prolongado, ficar de pé, alcançando coisas; ausência de variação postural; carga dinâmica como carregamento de pesos, transporte de pesos, flexão forçada, rotação do tronco, empurrar ou puxar cargas. Para os fatores ambientais, estão as vibrações, escorregar, cair, e tarefas do trabalho como monotonia, repetição, insatisfação. Listou 55 fatores individuais entre os quais a idade, 0 sexo, a forma física, a mobilidade de coluna, os fatores genéticos, a estrutura postural, a escoliose grave, a diferença no comprimento dos membros inferiores, as várias alterações degenerativas de coluna, os hábitos de fumar e ingerir álcool, os vários antecedentes médicos sociais e psicológicos.

WICKSTROM \& PENTTI (1998) acompanharam durante 24 meses, 117 trabalhadores de escritório e 189 de produção de duas indústrias (uma de construção naval e outra de componentes de ventiladores) e consideraram os fatores físicos (ruído, frio, lay-out, baixa iluminação), carga biomecânica (erguer, carregar, empurrar, puxar; posturas "desajeitadas"; postura de pé arriscadas e posturas sentadas arriscadas) e fatores psicossociais (poucas possibilidades de alte- 
rar as condições de trabalho, falta de reconhecimento e respeito no trabalho; fatores imprevistos no trabalho; estresse) e encontraram que exposição a fatores biomecânicos para trabalhadores de escritório e de produção, estresse para trabalhadores de escritório e fatores imprevistos para os de produção, foram os preditivos de lombalgia. Para absenteísmo e lombalgias recorrentes, cargas biomecânicas foram tidas como um dos fatores preditivos.

DEMPSEY e colaboradores (1997) sugerem que entre as variáveis individuais, devem ser consideradas: Idade - riscos de lombalgia tende a aumentar com idade e em algumas faixas, diminui; entretanto devem ser considerados possíveis fatores de confundimento relacionados com as exigências do trabalho e as exposições ocupacionais anteriores. Gênero: maior risco em mulheres (possível confundimento com diferentes exigências do trabalho). História de acidentes (história prévia de lombalgia aumenta significativamente o risco). Cuidados devem ser tomados ao medir risco futuro independente ou agravamento de um episódio prévio. Devem ser considerados a força muscular relativa (o aumento da força muscular relativa reduz riscos de lombalgias), o tabagismo, (fumar aumenta risco de lombalgias) e os fatores psicossociais cujo risco aumenta com certas variáveis e o possível confundimento com exigências do trabalho. Descartam os seguintes fatores: altura e peso, patologias, fatores genéticos, consumo máximo de oxigênio, força muscular absoluta.

VENNING e colaboradores (1987) estudando 4306 trabalhadores de enfermagem em hospitais gerais no Canadá durante um ano, encontraram uma taxa anual de lesões (acidentes) de 4,9\% (IC 4,4-5,7\%,95\%); as localizações foram de $60 \%$ para a região lombar, $18 \%$ dorsal e $16 \%$ cervical. Destes casos, $48 \%$ não perdeu nenhum dia de trabalho, 33\% ausentou-se de 1 a 15 dias e $19 \%$, mais de 15 dias. Quatro fatores encontrados foram significativos $(p<0,01)$ preditivos de lesão de coluna: setor de atividades, levantamento de cargas, função de trabalho e lesão prévia de coluna. Os dados obtidos indicam que as características do trabalho, mais que as características pessoais, são preditivos maiores de lesões de coluna entre enfermeiros.

RIIHIMAKI (1991) descreve os seguintes fatores de risco relacionados ao trabalho:

- Trabalho físico intenso - embora vários estudos não detalhem minuciosamente o que se conceitue por trabalho físico pesado, sendo mais baseado numa impressão geral ou alguns não detectem uma correlação significativa ou ainda, outros se baseiem na percepção subjetiva (que pode ser influenciada pela dor), vários estudos indicam que degenerações radiograficamente detectáveis estão relacionadas ao trabalho pesado.

- Levantamento, carregamento, puxar e empurrar cargas pesadas

- Curvar, rotação, flexionar, ou outras posturas de coluna não "neutras" - uma das dificuldades de se avaliar este tipo de posturas, é que em geral encontra-se associada ao deslocamento de cargas. No estudo feito pelo autor com seus colaboradores com empregados de escritório que relataram posturas em flexão ou curvadas, observou-se uma correlação positiva com ciatalgia, sendo que estes raramente realizavam carregamento de pesos.

- Conduzir veículos automotores - Existem evidências de que se trata de atividade com risco para lombalgia, ciatalgia, e hérnia de disco. Também encontra-se um aumento de prevalência de degenerações de coluna, radiograficamente detectáveis.

- Posição sentada - Os estudos são controversos, sendo que vários não indicam aumento de risco, enquanto outros indicam um risco aumentado para lombalgias, em trabaIhadores que permanecem sentados por longos períodos ou sedentários. Embora as evidências sejam contraditórias, o autor considera que seja biologicamente possível, pois tem sido demonstrado que a pressão intradiscal encontra-se aumentada na posição sentada, particularmente em posição ereta e sem suporte. Também é possível que a posição prolongada leve a efeitos deletérios na nutrição dos discos intervertebrais.

- Trauma - Num estudo prospectivo realizado por LLOYD \& TROUP (1983) observouse que num terço dos episódios de lombalgia houve um verdadeiro acidente, em um quinto, uma lesão não acidental e para os outros casos, não se encontrou qualquer evento diretamente relacionado à lesão. Os acidentes com lesão, em especial as quedas, têm pior prognóstico.

- Fatores psicossociais. Vários fatores têm 
sido relacionados ao trabalho, tais como a monotonia, insatisfação no trabalho e associados às lombalgias.

Num estudo prospectivo em 3000 empregados de aviação, fatores psicossociais no trabalho foram considerados melhores preditivos de lombalgias futuras do que os achados físicos. (BIGOS et al sem ano). Entretanto FEYER e colaboradores (1992) estudando dois grupos de trabalhadores (carteiros e enfermeiros) e um grupo de pacientes, não encontraram relação entre insatisfação no trabalho e lombalgias, nem associação entre variáveis psicológicas e lombalgia; consideram que a natureza dos fatores psicológicos permanece obscuro. $O$ que notam, concordante com a literatura, é a presença de alterações nos escores de testes psicológicos em pacientes lombálgicos crônicos.

SYMONDS e colaboradores (1996) encontraram, entre trabalhadores de escritório e também nos de produção, que apresentaram absenteísmo mais prolongado por lombalgia, atitudes e crenças mais negativas, quando comparadas com os que eram sintomáticos ou assintomáticos mas sem afastamentos mais prolongados.

MAIRIAUX (1988) sugere três fatores de risco principais, as sobrecargas mecânicas excessivas ou repetidas, onde a primeira crise álgica ocorre mais precocemente nos expostos (1), o aumento de sintomas mais graves e a incapacidade ao trabalho se mantém mais prolongada; a rigidez postural (2); as vibrações de corpo inteiro (3).

SHELERUD (1998) lista o trabalho físico intenso, as posturas de trabalho estáticas, 0 levantamento de pesos, as atividades com flexão e rotação de coluna, e as vibrações de corpo inteiro e cita outros autores, como ilustra o quadro a seguir.

Quadro2 Prevalência de lombalgia em estudos comparativos de populações trabalhadoras executando trabalhos pesados e leves.

\begin{tabular}{|l|c|c|}
\hline autor e ano & trabalho pesado (\%) & trabalho leve (\%) \\
\hline HULT (1954) & & \\
a) lombalgia & 64,5 & 52,7 \\
b) lombalgia grave & 10,6 & 6,8 \\
c) absenteísmo & 43,5 & 22,5 \\
\hline LAWRENCE (1955) & 41 & 29 \\
\hline ROWE (1969) & 47 & 35 \\
\hline IKATA (1965) & 22,4 & 5,2 \\
\hline MAGORA (1970) & 21,6 & 10,4 \\
\hline LLOYD (1986) & 69 & 58 \\
\hline
\end{tabular}

\section{Instrumentos \\ metodológicos nos estudos das lombalgias e dos fatores ocupacionais}

SCHIERHOUT e colaboradores (1994) estudando instrumentos para avaliação de cargas físicas, mais especificamente em relação aos fatores posturais, consideram quatro diferentes enfoques, os questionários, os checklists, os métodos de observação e as medições diretas. Nesta citação, em ordem crescente, temos aumento de custos e de especificidade e redução de capacidade, versatilidade e generalidade.
DEMPSEY (1998) realizou uma revisão dos principais critérios relacionados aos limites aceitáveis para a realização de atividades físicas. São os critérios biomecânicos, fisiológicos, e psicofísicos. Na abordagem biomecânica, a meta é projetar tarefas cuja capacidade músculo esquelética não seja excedida. Os critérios mais comuns são limites de compressão discal L4/L5 ou $L 5 / s 1$ e o torque máximo para as articulações. Os estudos são experimentais e modelos são construídos para estimar a aceitabilidade da tarefa. Na abordagem fisiológica as tarefas são projetadas para que as respostas fisiológicas estejam dentro de limites aceitáveis. As 
respostas mais estudadas são a fadiga local ou geral e os critérios mais comuns são o consumo energético, avaliado através do consumo de oxigênio nos parâmetros da normalidade. Na abordagem psicofísica a meta é prescrever tarefas que sejam aceitáveis para a maioria dos trabalhadores, com adequação de peso, forças, frequência máxima aceitável para certas tarefas. Os experimentos costumam ser feitos em laboratórios e modelos são construídos para valores preditivos para tarefas variáveis e trabalhadores. $O$ papel da epidemiologia consiste em (1) determinar/verificar a significância "etiológica" de uma determinada variável; (2) verificar a validade de determinados critérios, estabelecendo a relação entre uma variável específica e a probabilidade de dano, incapacidade ou outro efeito; (3) providenciar orientação no desenvolvimento de critérios através de considerações de relações entre uma determinada variável e resultados medidos previamente selecionados.

LAVANDER e colaboradores (1999) num estudo metodológico empírico, aplicaram cinco distintas técnicas em 93 postos de trabaIho, de 178 trabalhadores, para avaliar os riscos de lombalgia no trabalho, (1) 0 Revised National Institut of Occupational Safety and Health (NIOSH) Work Practices Guidelines for Manual Lifting, (2) O Tridimencional Static Strength Prediction Program (SSPP), (3) O Lumbar Motion Monitor (LMM) e $(4,5)$ duas variações de check-lists -Risk Factor Checklist (RFC), da United Autoworkers. Os resultados mostraram que os resultados dependem do método utilizado para a avaliação. O SSPP parece ser mais "conservador" pois a maioria dos postos foi considerada de baixo risco. $\mathrm{O}$ modelo da $\mathrm{NIOSH}$, ao contrário, classificou a maioria dos postos de alto risco. Entre ambos, RFC e o LMM, sendo que o LMM indica maior risco que o RFC. Os viezes de classificação observados são parcialmente atribuídos à natureza das atividades executadas. Os métodos NIOSH E LMM são mais apropriados para carregamento de pesos. O SSPP é pouco sensível às atividade de levantamento de pesos e mais apropriada para determinar o risco de lesões agudas (acidentes). Os métodos LMM e NIOSH são mais apropriados para determinar os riscos de Lesões por Esforços Repetitivos (LER/DORT). A concordância entre os métodos para priorizar as inter- venções ergonômicas foi de modo geral baixo.

BONGERS e colaboradores (1993) revisaram os estudos epidemiológicos que tratavam de aspectos psicossociais ocupacionais descritos como associados aos sintomas músculo esqueléticos e encontraram 44 estudos transversais e 15 longitudinais. Destes, somente 29 transversais e 3 longitudinais analisaram especificamente a influência de fatores psicossociais no trabalho. Do conjunto somente 22 ajustaram de alguma forma, para a sobrecarga física. Citam o "Inventário" Multifásico de Personalidade Minnesota, que considera cinco categorias: (1) as demandas e controle no trabalho, (2) o suporte social, (3) as características individuais, (4) os sintomas de estresse e (5) os indicadores de saúde física e comportamental. Os estudos apresentaram dados contraditórios de correlação entre os aspectos psicossociais e doença e dificuldades para medir as variáveis dependentes e independentes relacionadas com a carga física. Entretanto, concluíram que o trabalho monótono, a alta carga física percebida e a pressão de tempo estão relacionados com sintomas músculo esqueléticos.

BURDOF (1992) realizou um levantamento a partir da base de dados Medline, 19811990, selecionando 104 publicações de estudos epidemiológicos, com medidas de exposição a fatores de risco ocupacionais para problemas lombares. Foram excluídas 23 referências, que tratavam de revisões e de estudos secundários. Foram 81 artigos originais numa primeira seleção. Foi feita uma segunda seleção, cujos critérios foram dados numéricos completos dos fatores de risco e dados de exposição. Assim 34 trabaIhos foram analisados, para avaliar os métodos de exposição a fatores específicos dos ambientes de trabalho. Nestes, 33\% foram avaliados através de questionários, $9 \%$ através de observações e $5 \%$ através de medições diretas.

AGIUS e colaboradores (1994) construíram um questionário que permitisse a identificação e avaliação de gravidade de lombalgias para fins epidemiológicos e para sua validação, aplicaram em uma população trabalhadora em minas de carvão ( $n=471)$. O questionário consta de 12 questões para dor, irradiação, frequência, gravidade, relação com dificuldades para tarefas 
específicas, interferência com o trabalho normal e afastamentos do trabalho. As respostas foram parcialmente validadas através da comparação com os atestados médicos de mais de dois dias, cujas causas foram atribuídas à lombalgia. Em trabalhadores que referiram lombalgia nos 12 meses anteriores, para as questões relativas aos afastamentos por causa de lombalgia, a sensibilidade foi de $82 \%$ e a especificidade, de $84 \%$.

\section{Técnicas observacionais posturais}

GENAIDY \& AL-SHEDI \& KARWOWSKI (1994) discutindo a análise postural consideram as posturas do corpo nas técnicas observacionais por percepção visual, usando duas abordagens, a primeira através de observação direta do trabalho e a segunda através do vídeo, onde a postura é determinada analisando-se posteriormente o registro. O registro pode ser contínuo ou intermitente. A classificação postural é feita em papel ou por computador. A classificação postural utilizada em técnicas observacionais podem ser categorizadas em macropostural, cujo primeiro sistema de classificação foi desenvolvido por KARHU e colaboradores em 1977, conhecido como Ovako Working Postures Analysing (OWAS) e depois completado por outros autores $\mathrm{e}$ micropostural descrita por KILBOM (1986) e ARMSTRONG (1982) e depois complementado por outros numerosos autores, que descrevem com grande detalhe as posturas não neutras e a classificação de atividades postura-trabalho, desenvolvidas por FOREMAN \& TROUP em 1987, BATY (1986) e RYAN (1989). Outras técnicas observacionais são citadas, como o de PRIEL (1974), que define as posturas do trabalhador numericamente, cujo esquema de registro foi denominado de "posturegram". Ainda citam o AET - Arbeitswissenschaftliches Erhebungsverfahren zur Tatigkeitsanalyse (ROHMERT \& LANDAU, 1983) e o ARBAN (HOLZMANN, 1982)

KILBOM (1994) realiza um estudo revisando os principais métodos observacionais que permitem avaliar a 'exposição' física. A principal mensuração quantitativa de exposição é a duração ou frequência de certas posturas ou atividades, durante um certo tempo.
Um dos mais "simples" é feito com a observação direta, lápis e papel, considerando certos intervalos de tempo. Outros, registram informações através de padrões posturais prévios. Ainda existem sistemas e aparelhos, tipo PEO (Portable Ergonomic Observation), onde os registros são complementados por entrevistas e todas as tarefas mais importantes numa semana são anotadas, com sua duração. Vídeos acoplados a computadores programados para registros em tempo real também são descritos, para avaliar a frequência e duração de movimentos e posturas. Um método bastante descrito foi o OWAS, Ovako Work Posture Analyzing System, utilizado particularmente em estudos ergonômicos, para identificar e avaliar posturas desconfortáveis no trabalho. As posições lombares e de membros são observadas e registradas para 84 diferentes posturas definidas por este método. As tarefas específicas são identificadas para cada trabalho em separado, os trabalhadores, observados durante dois períodos de 10 minutos, englobando todas as tarefas importantes. O procedimento consiste em 60 observações de cada trabalhador, por intervalos de 20 segundos. Este método por amostragens permite incluir todas as atividades relevantes.

BURDOF \& LAAN (1991) estudaram 35 trabalhadores de manutenção de uma empresa de fundição na Holanda, numa avaliação de exposição às sobrecargas posturais de coluna. Utilizaram três distintos métodos para o estudo, sendo o primeiro um questionário sobre as cargas do próprio trabalho, considerando a duração e frequência das posturas habituais de trabalho, as movimentações, atividades de carregamento, movimentos específicos da coluna lombar em uma jornada de trabalho. $O$ segundo foi feito com as mesmas questões básicas, mas através de um registro pelo próprio trabalhador de um "diário", com anotações a cada hora, em uma jornada completa. O terceiro foi o Ovako Working Posture Analyzing System - OWAS. $O$ estudo todo foi feito em três semanas.

BURDOF (1992) considera seis fatores de risco ocupacional para lombalgias sendo o trabalho físico pesado (1), postura estática no trabalho (2), inclinar e agachar frequentemente (3), carregar e movimentos com esforços (4), movimentos repetitivos (5), e vibrações de corpo inteiro (6). Para estes fatores, levanta sete técnicas observacionais 
(número de posturas estáticas mantidas por ao menos 30 segundos, por jornada de trabalho, porcentagem do tempo de trabalho sem movimento, número de mudanças posturais por minuto, frequência de flexão e curvar, levantamento de cargas, movimentos com esforços e movimentos repetidos) e quatro métodos diretos (eletromiografia, flexão da coluna medindo ângulo médio, velocidade angular; pelo método $\mathrm{NIOSH}$, peso, frequência de levantamentos, localização vertical, e distância de deslocamento vertical); mensuração de vibrações (acelerômetros) para medir a exposição aos fatores de risco para lombalgias ocupacionais.(NIOSH). Considera que a avaliação de exposição em epidemiologia ocupacional para lombalgias necessita de maior atenção. A caracterização de exposição de carga postural para coluna deve levar em consideração a frequência e duração do esforço postural e de movimentos, sua variação durante atividades laborais com a jornada e em diferentes jornadas, e as diferenças entre trabalhadores realizando as mesmas tarefas.

BURDOF apresenta em 1993, fontes de vieses metodológicos relacionados com a estimativa de risco devido à variabilidade da exposição a cargas posturais para coluna lombar, em diferentes grupos ocupacionais; a taxa de classificação inadequada para exposição a flexão do tronco e rotação, variam de 0,03 a 0,35. Altas taxas de classificações inadequadas podem levar facilmente ao viés do risco estimado maior que $50 \%$.

\section{Uma breve discussão}

FRANK e colaboradores (1995) descrevem as três abordagens mais comuns para as lombalgias ocupacionais, relacionadas com a etiologia e o seu prognóstico. (1) O olhar da patologia clínica, que privilegia a doença e seu prognóstico, sem grande preocupação com a etiologia e sua prevenção; neste caso a incapacidade advém de lesões mais graves, tais como hérnia de disco ou complicações psicopatológicas secundárias, tais como a depressão ou as desordens psicossomáticas; (2) O olhar da exposição biomecânica, que considera a lombalgia e suas limitações decorrentes da exposição biomecânica, que excessiva e repetidamente atuam sobre os te- cidos da coluna; neste caso, a dor e a incapacidade seriam preveníveis através de intervenções nos postos de trabalho, para corrigir os problemas ou através de legislações específicas para as questões ergonômicas nos locais de trabalho. (3) O incentivo, advindo da própria estrutura social, que combina insatisfação com o trabalho, e os benefícios das compensações por incapacidade no trabalho e do Seguro Social.

O grupo PARIS TASK FORCE ON BACK PAIN (2000) observa que a literatura em epidemiologia da história natural de lombalgias tem sido largamente limitada em si mesma para as incapacidades e absenteísmo no trabalho. A correlação considerada pobre entre lombalgias e as incapacidades às atividades laborais podem ocorrer parcialmente devido à ausência de um "paradigma" apropriado. Colocam duas questões que podem ilustrar bem o problema. A primeira é que alterações lombálgicas, sem limitação ao trabalho é altamente prevalente na indústria. Para RIIHIMAKI (1991) a maioria dos estudos tem sido retrospectivos, assim é difícil de determinar se os aspectos psicossociais seriam antecedentes ou consequentes à lombalgia. É difícil de determinar se estes fatores têm um papel na etiologia das lombalgias ou se somente afetam a percepção ou o comportamento do doente.

CROFT (1996) citado por ABEHAIM (2000) encontrou que somente $19 \%$ dos trabalhadores em atividade e portadores de lombalgia procuraram médico no período de um ano. As alterações lombálgicas levam a mudanças dinâmicas na interação trabalhador-trabalho. Assim, trabalhadores em atividades de "alto risco" apresentam menos lombalgia que os que se encontram nos de "baixo risco". Alguns estudos qualitativos revelam que trabalhadores podem ser realocados em diferentes tipos de trabalho, quando apresentam lombalgia. Assim, diferentes atividades/funções podem estar associados não somente com diferentes tipos de fatores de riscos ergonômicos, mas também com diferentes grupos de trabalhadores, com diferentes histórias de lombalgia!

DERRIENNIC (1990) observa que na França, numa mesma empresa os postos de trabalho não se distribuem de forma aleatória, parecendo existir um "gerenciamento de ida- 
des". Na indústria automobilística, 75\% dos trabalhadores de uma linha de montagem e $66 \%$ dos prensistas tinham menos de 40 anos e nas empresas de montagem de peças eletrônicas só $10 \%$ tinha mais de 30 anos. Em outro estudo, retrospectivo de 5000 trabalhadores de uma indústria automotiva, observou-se que as tarefas fisicamente mais exigentes são executadas pelos mais jovens e as mais leves pelos mais idosos, independentemente dos afastamentos por doença e da qualificação; ao analisar as mudanças de postos (de trabalho pesado para leve) ocorridas em 5 anos, observou-se que os que mudaram foram os mais velhos. Em grandes inquéritos nacionais franceses sobre condições de trabalho, tem-se observado que as distribuições por idades e por atividade econômica não são homogêneas e algumas distribuições mantêm-se constantes no tempo.

FRANK e colaboradores (1995) levantam cinco temas desafiadores para as futuras pesquisas sobre as lombalgias ocupacionais. São eles:

1. Definindo lombalgia: qual definição de caso estudado?

2. Medindo lombalgia: pode ser validada e medida a confiabilidade?

3. Lesão ou doença: trata-se de um evento agudo, uma condição recorrente ou de uma doença industrial?

4. Distinguindo etiologia de fatores prognósticos de risco: qual a limitação dos dados descritivos (cross-sectional data) e qual é o melhor desenho de estudo?

\section{Referências bibliográficas}

ABENHAIM,L; SUISSA, S.; ROSSIGNOL, M.- Risk of recurrence of occupational back pain over three year follow up.British Journal of Industrial Medicine,45, 829-833, 1988.

ABENHAIM,L.; ROSSIGNOL, M.; VALAT, J.P; et al (PARIS TASK FORCE ON BACK PAIN) .- The role of activity in the therapeutic Management of Back Pain.Spine, 25,4, 1-335, 2000.

AGIUS, R.M.; LLOYD, M.H.; CAMPBELL, S.; et al.- Questionnaire for the identification
5. Avaliando a exposição: qual seria o intervalo de tempo de exposição adequado a ser obtido? E como poderia ser mais abrangente a lista de variáveis? Segundo estes autores, raros são os trabalhos que contemplam os fatores de forma equilibrada; consideram então que esta compartimentalização disciplinar e as dificuldades metodológicas inerentes às pesquisas sobre lombalgias ocupacionais contribuem para criar polêmicas que dificultam o progresso na identificação e as ações sobre as causas modificáveis do aparecimento das lombalgias e a prevenção de sua incapacidade.

Mairiaux (1988) considera dois níveis de atuação, um sobre a população geral, desenvolvendo uma política de saúde pública favorecendo o estado físico e a redução do tabagismo e de outra parte, para os trabalhadores das empresas, da implantação de programas de prevenção que inclua uma vigilância epidemiológica simples mas contínua, da prevalência de sintomas álgicos de coluna; a identificação de funções e postos de trabalho de risco, a partir de dados epidemiológicos e da análise de acidentes de trabalho; a análise ergonômica de exigências nos postos sob risco e as ações de prevenção específicas, combinando numa proporção variável, função da natureza das exigências, informação e formação dos trabalhadores e concepção ergonômica de equipamentos. of back pain for epidemiological purposes.- Occupational and Environmental Medicine, 51, 756760, 1994.

BEHRENS, V.; SELIGMAN, P.; CAMERON, L. et col.- The prevalence of back pain, hand discomfort and dermatitis in the US Working Population.- American Journal of Public Health, 84, 11, 1780-1785, 1994.

BONGERS, P.M.; WINTER, C.R.; KOMPIER, M.A.J.; HILDEBRANDT, V.H.- Psychosocial 
Factors at work and Musculoskeletal Disease.- Scandinavian Journal of Environmental Health 19:297-312, 1993.

BORKAN,J. ;REIS,S.; HERMONI, D.;BIDERMAN,A. - Talking About The Pain : a patient-centered study of Low Back Pain in Primary Care. Society Science and Medicine, 40 (7) : 977 -988, 1995.

BRUN, V.; SIMON, L.- Les principes de rééducation des lombalgiques. Revue du Praticien,36, 13,1986.

BURDOF, A.- Comparison of methods for the assessment of postural load on the back.Scandinavian Journal of Work and Environmental Health, 17:425429, 1991.

BURDOF, A.- Exposure assessment of risk factors for disorders of the back in occupational epidemiology.- Scandinavian Journal of Work and Environmental Health, 18:1-9, 1992.

BURDOF, A.- Bias in risk estimates from variability of the exposure to postural load on the back in occupational groups.Scandinavian Journal of Work and Environmental Health, 19:1950,1993

CASSOU,B.; DERRIENNIE， F.; IWATSUBO,Y.; AMPHOUX, M. Physical disability after retirement and occupational risk factors during working life: a cross sectional epidemiological study in the Paris area. Journal of Epidemiology and Community Health 46 : 506$511,1992$.

CHIRON, M.- Cahiers de Notes Documentaires, 111, 1983, p.167176.

CLINICAL STANDARDS ADVISORY GROUP - Review Essay Back Pain and Epidemiology Review : The Epidemiology and Cost of Back Pain.- Society, Science and Medicine, 42(4) : 561 - 563,1996
DEMPSEY, G. P.; BURDOF, A.; WEBSTER, B.S.- The Influence of Personal Variables on Work-Related Low-Back Disorders and Implications for Future Research.Journal of Occupational and Environmental Medicine, 39, 8, 748-759, 1997.

DEMPSEY, P.G.- A critical review of biomechanical, epidemiological, physiological and psychophysical criteria for designing manual materials handling tasks.- Ergonomics, 42, 1:73-88, 1998.

DERRIENNIC,F. - Une problématique difficile: I'association travail-vieillissement.- La Revue du Praticien, 115 : 91 99,1990 .

DERRIENNIC, F . ;IWATSUBO, Y .; MONFORT,C.; CASSOU,B. - Evolution of osteoarticular disorders as a function of past heavy physical work factors: longitudinal analysis of 627 retired subjects living in the Paris area.- British Journal of Industrial Medicine,50:851-860, 1993.

FEYER, A.M.; WILLIAMSOM, A.; MANDRIK, J.; SILVA, I.; HEALY, S.- Role of psychosocial risk factors in work-related low-back pain.- Scandinavian Journal of Work and Environmental Health, 18, 368-375, 1992.

FINOCCHIARO, J.; ASSAF, D.L.Infortunística das Lombalgias na Construção.- Revista Brasileira de Saúde Ocupacional, p 40-51.

FRANK, W.; PULCINS, M.A.; KERR, M.S ET AL.- Occupational back pain - an unhelpful polemic.- Scandinavian Journal of Work and Environmental Health, 21, 3-14, 1995.

GAUDEMARIS, R.; BLATIER,J.F.; QUINTON, D.; et al. - Analyse du Risque lombalgique en milieu professionnel.- Revue Epidemiologie et Santé Publique, 34,308-317, 1986. 
GENAIDY, A.M.; AL-SHEDI, A.A.; KARWOWSKI, W.- Postural Stress Analysis in Industry.- Applied Ergonomics, 25,2:77-87, 1994

GRANIER, M.; TRIGALOU, I.; HUGELE, B.; PLUOT, J.- Lombalgies dans la fonction publique territoriale.- .- Archives des Maladies Professionnelles, 59, 2, 103-109, 1998.

GUO, H-R; TANAKA, S.; CAMERON, L.L. ET AL.- Back pain among workers in the United States: National estimates and workers at high risk.- American Journal of Industrial Medicine., 28:591-602, 1995.

GYNTELBERG,F.- One-year incidence of low back pain among male residents of Copenhagen aged 40-59. Danish Medical Bulletin, 21:30, 1974.

HALES, T.R.; BERNARD, B.P.- Epidemiology of Work related musculoskeletal disorders.Orthopedic Clinics of North America, 27,4: 679-709, 1996.

HARBER., P.; BILLET, E.; GUTOWSKI, M. E al.- Occupational Low- Back Pain in Hospital Nurses.- Journal of Occupational Medicine, 27,7, 518-524, 1985.

HARDLER, N.M.- A Rheumatologist's View of the Back.- Journal of Occupational Medicine, 24,4,282-285,1982.

HASHEMI, L.; WEBSTER, B.; CLANCY, E.A; VOLINN, E.- Length of disability and cost of workers' compensation low back pains claims.- Journal of Occupational and Environmental Medicine,39, 10, 937-945, 1997.

HELIOVAARA, M.; IMPIVAARA, O.; SIEVERS, K. Et al.- Lumbar disc syndrome in Finland.- Journal of Epidemiology and Community Health, 41,251$258,1987$.

HYYTIAINEN, K.- Attitudes towards prevention of low back disorders in industry.Occupational Medicine, 44:83-86, 1994.
KATZ, R.T.; RONDINELLI, R.D.- Impairment and Disability Rating in Low Back Pain.Occupational Medicine, 13, 1 213230, 1998.

KELSEY, J.L.; GOLDEN, A.L; MUNDT, D.J.Low Back Pain/prolapsed lumbar intervertebral disc.- Rheumatic Disease Clinics of North America, 16, 3, August 1990.

KILBOM, A.- Assessment of physical exposure in relation to work-related musculoskeletal disorders - what information can be obtained from systematic observation? Scandinavian Journal of Environmental Health, 20 special issue:30-45, 1994.

KNIBBE, J.J.; FRIELE, R.D.- Prevalence of back pain and characteristics of the physical workload of community nurses.Ergonomics, 39,2,186-198, 1996.

LAVANDER， S.; OLESKE， D.M.; $\mathrm{NICHOLSON,} \mathrm{L.} \mathrm{Etal.-} \mathrm{Comparison} \mathrm{of} \mathrm{five}$ methods used to determine Low Back Disorders Risk in a Manufacturing Environment.- Spine, 24, 14,14411448, 1999.

LEIGHTON, D.J.; REILLY, T.- Epidemiological aspects of back pain: the incidence and prevalence of back pain in nurses compared to the general population.- Occupational Medicine, 45,5, 263-267, 1995.

LEUNG, A.S.L; LAM, T-H.; HEDLEY, A.J.; TWOMEY, L.T.- Use of a subjective health Measure on Chinese Low Back Pain patients in Hong Kong.- Spine, 24,10, 961-966, 1999.

MACDONALD, M.J.; SOROK, G.S.; VOLINN, E ET AL.- A descriptive Study of recurrent low back pain claims. - Journal of Occupational and Environmental Medicine, 39, 1, 35-43, 1997.

MAIRIAUX, P.- Lombalgies en milieu du travail.- Archives des Maladies Professionnelles., 49,2, 85-95, 1988. 
RIIHIMAKI, H. - Low-back pain, its origin and risk indicators.- Scandinavian Journal of Work and Environmental Health,17:81-90, 1991.

SCHIERHOUT, G.H.; BRIDGER, R.S.; MYERS, J.E- Development of observational methods for estimation of exposure to workplace postural stress.- Occupational Medicine, 44,5, 262-266, 1994.

SHELERUD, R.- Epidemiology of Occupational Low Back Pain.- Occupational Medicine: State of the Art Review, 13, 1, 1-22, 1998.

STUBBS, D.A.; BUCKLE, P.W.; HUDSON, M.P. et al.- Ergonomics, 26,8,755$765,1983$.

SYMONDS, T.L.; BURTON, A.K.; TILLOTSON, KM et al.- Do attitudes and beliefs influence work loss due to low back trouble? Occupational Medicine 46,1:25-32, 1996.

TSAI, SP; GILSTRAP, EL; COWLES, SR et al.Personal and Job Characteristics of Musculoskeletal Injuries in an Industrial Population.- Journal of Occupational Medicine 34,6, 606-612, 1992.
VENNING, P.J.; WALTER, S.D.; STITT, L.W.Personal and Job-related Factors as Determinants of Incidence of Back Injuries among Nursing Personnel.- Journal of Occupational Medicine, 29, 10, 820-825, 1987.

VIGNON, G.; MASSET, J.C.- Evolution au bout de 10 ans de 51 lombalgiques accidentés du travail.- Bulletin de Medecine Legale et Toxicologie, 22,3, 323-328, 1979.

WADDELL, G; BURTON, AK- Occupational health guidelines for the management of low back pain at work: evidence review.Occupational Medicine, 51,2,124135,2001 .

WEBSTER, B.S.; SNOOK, S.- The Cost of Compensable Low Back Pain.- Journal of Occupational Medicine, 32, 1, 1990, p.13-15.

WICKSTROM, G.J; PENTTI, J.- Occupational Factors affecting sick leave attributed to low-back pain. Scandinavian Journal of Work and Environmental Health, 24,2:145-152, 1998. 